Bull. Mater. Sci., Vol. 35, No. 4, August 2012, pp. 601-609. (C) Indian Academy of Sciences.

\title{
Percolation based enhancement in effective thermal conductivity of HDPE/LBSMO composites
}

\author{
MADHUSREE KOLE, D TRIPATHI and T K DEY* \\ Cryogenic Engineering Centre, Indian Institute of Technology, Kharagpur 721 302, India
}

MS received 8 March 2011; revised 30 November 2011

\begin{abstract}
Thermal conductivity of composites with electrically conducting $\mathrm{La}_{0.7} \mathrm{Ba}_{0.15} \mathrm{Sr}_{0.15} \mathrm{MnO}_{3}$ (LBSMO) filler of nanometric grain size in HDPE matrix is investigated. Volume fraction of LBSMO fillers was varied between 0 and $\mathbf{0 \cdot 3 0}$. SEM photographs of the composites show the presence of clusters and percolative paths, particularly for composites prepared with higher filler volume fractions. The effective thermal conductivity of the composites displays significant enhancement with increasing filler content in HDPE. A maximum enhancement of $\sim 65 \%$ compared to that for pure HDPE has been observed for composite with 0.30 volume fraction of LBSMO filler. Most of the models those are generally used to predict the properties of two phase mixtures, has been found either to under/overestimate the measured effective thermal conductivity of the composites. We confirm that the observed rapid increase in the effective thermal conductivity of HDPE/LBSMO composite over the studied range of filler volume fraction (viz. 0-0.30), is predicted very well, considering the effect of percolation as proposed by Zhang et al (2009).
\end{abstract}

Keywords. Thermal conductivity; HDPE/La $\mathrm{La}_{0.7} \mathrm{Ba}_{0.15} \mathrm{Sr}_{0.15} \mathrm{MnO}_{3}$ composites; transient plane source (TPS); percolation model.

\section{Introduction}

Over the last few decades, the microelectronic devices have been developed with significantly higher integration density, faster performance and miniaturization, but with penalty of larger and larger power density. As a result, in order to sustain the growth in microelectronic systems, development of superior heat-dissipating materials/composites has become important. Polymer filled with appropriate fillers having improved thermal conductivity offers possibilities of its applications in the thermal management of microelectronic devices. Investigations on the thermophysical properties of polymers containing various kinds of metallic and ceramic fillers, including carbon nanotubes, have been widely reported (Wong and Bollampally 1999; Xu et al 2001; Kumlutas et al 2003; Goyal et al 2007). It is now known that the thermal properties of particles filled polymers is a complex function of their geometry and thermal conductivity of the different phases, distribution of the fillers in the matrix, contact between the particles and several models have been proposed so far. However, in spite of this, accurate prediction of effective thermal conductivity of composites still remains a challenge and consequently measurement of thermal conductivity of the newer composites is important.

Perovskite manganese oxides, with mixed valence states of manganese ions $(+3$ or +4$)$, obtained by divalent or monovalent doping in place of rare-earth ion, are known to be materials of scientific interest due to their rich electric

*Author for correspondence (tapasdey @ hijli.iitkgp.ernet.in) and magnetic phase diagram associated with their 'colossal magneto-resistance' (CMR) properties. These materials exhibit a transition from paramagnetic insulating state to ferromagnetic metallic state as the temperature is decreased. Thermal conductivity of lanthanum manganites have been reported earlier (Hejtmanek et al 1999; Fujishiro and Ikebe 1999; Cohn 2000; Battabyal and Dey 2006) and it is known that the heat conduction in manganites is primarily phonon dominated and the electronic part contributes only $\sim 1 \%$ to the total thermal conductivity $(\sim 2.5 \mathrm{~W} / \mathrm{mK})$. In the present communication, we report our results on the effective thermal conductivity of $\mathrm{HDPE} / \mathrm{La}_{0.7} \mathrm{Ba}_{0.15} \mathrm{Sr}_{0.15} \mathrm{MnO}_{3}$ (LBSMO) composites. To our knowledge, thermal properties of high density polyethylene containing lanthanum manganites as fillers have not been reported previously. The effective thermal conductivity as a function of filler (LBSMO) volume fraction between 0 and 0.3 is presented and application feasibility of various models have been discussed. We confirm that percolation model gives a superior prediction of the experimental data in the studied range of LBSMO filler content in HDPE.

\section{Thermal conductivity models for two phase systems}

Over the years, several theoretical models have been proposed to predict the effective thermal conductivity of twocomponent systems (solids and fluids). These models are either derived theoretically or include one or more experimentally determined/empirical parameters. Factors, such as, 
filler loading, particle size or shape, and homogeneity of the dispersed phase in the matrix, etc were taken into account in heat conduction models. The review and derivation of these models are out of the scope of the present study and only a brief summary on some of the important models is given. Excellent reviews of these models have discussed their applicability in two component solids and fluids (Progelhof et al 1976; Ott 1981; Keblinski et al 2002; Wang and Mujumdar 2007; Murshed et al 2008; Li et al 2009). Table 1 gives the expressions derived by a few widely referred models for composites.

Solving Laplace's equation and assuming absence of any interactions between the filler particles, Maxwell (1954) was the first to calculate the effective thermal conductivity of random distribution of spheres in a continuous medium. Hamilton and Crosser (1962) later extended Maxwell's model to include an empirical factor, $n$, to account for the shape of the particles $(n=3$ for spheres and $=6$ for cylinders). It may be noted that neither Maxwell, nor, Hamilton and Crosser model contain any dependence on particle size, and they also imply that the temperature dependence of thermal conductivity is approximately the same as that of the matrix. Starting with Maxwell model (1954), Bruggeman (1935) derived another exact model for the effective thermal conductivity under different assumptions for permeability and field strength. A useful model for high filler-loaded composites was first suggested by Meredith and Tobias (1962) with a reasonable success. Cheng and Vachon (1990) assumed a parabolic distribution of the discontinuous phase in a continuous medium and determined the parabolic distribution constants by analysis, which was related to the discontinuous phase volume fraction. The effective thermal conductivity was then derived for the two-phase solid mixture. Using the concept of mean field, Bruggeman (1935) was the first to propose the effective medium theory (EMT) for electrical conductivity and dielectric constant, which was later extended for thermal conductivity of composites (Hakansson and Ross 1990; Kutcherov et al 1991; Hui et al 1999; Carson et al 2005). A semi-empirical model was reported by Lewis et al (1970), which was based on modification of the Halpin-Tsai equation (Meredith and Tobias 1962; Sass et al 1971). Their proposition takes into account effect of shape and orientation of the particle or, the type of packing for a two-phase system. The constant $A$, in their equation, takes care of the particle shape and their orientation with respect to the direction of the heat flow. The type of packing is accounted for by the term $\phi_{\mathrm{m}}$, viz. for randomly packed spherical particles, $A=1.5$ and $\phi_{\mathrm{m}}=0.637$; whereas for randomly packed aggregates of sphere or for randomly packed irregularly shaped particles, $A=3$ and $\phi_{\mathrm{m}}=0.637$. This model has been reported to be more successful for low and medium filler loaded composites $(<20 \%)$. Agari and Uno (1986) also proposed another semiempirical model, which is based on the argument that the enhanced thermal conductivity of high filler loaded composites originates from forming conductive channels or, chains of fillers. Their expression contains two constants, $C_{1}$ and $C_{2}$, which are experimentally determined and cannot be precisely predicted. All these models mentioned above, consider uniformly and periodically distributed filler particles and therefore, cannot reflect actual microscopic irregularities of the filler shape and distribution for composites with high

Table 1. Model predictions for effective thermal conductivity of particle filled polymers.

Model Prediction Eqn. no.

Meredith and Tobias (1962)

$\frac{\lambda_{\text {eff }}}{\lambda_{\mathrm{m}}}=\frac{2\left(2+\lambda_{\mathrm{f}} / \lambda_{\mathrm{m}}\right)-2\left(1-\lambda_{\mathrm{f}} / \lambda_{\mathrm{m}}\right) \phi}{2\left(2+\lambda_{\mathrm{f}} / \lambda_{\mathrm{m}}\right)+\left(1-\lambda_{\mathrm{f}} / \lambda_{\mathrm{m}}\right) \phi} \times \frac{(2-\phi)\left(2+\lambda_{\mathrm{f}} / \lambda_{\mathrm{m}}\right)-2\left(1-\lambda_{\mathrm{f}} / \lambda_{\mathrm{m}}\right) \phi}{(2-\phi)\left(2+\lambda_{\mathrm{f}} / \lambda_{\mathrm{m}}\right)+\left(1-\lambda_{\mathrm{f}} / \lambda_{\mathrm{m}}\right) \phi}$

Russell (1935)

$\lambda_{\text {eff }}=\lambda_{\mathrm{m}}\left[\left\{\phi^{2 / 3}+\frac{\lambda_{\mathrm{m}}}{\lambda_{\mathrm{f}}}\left(1-\phi^{2 / 3}\right)\right\} /\left\{\phi^{2 / 3}-\phi+\frac{\lambda_{\mathrm{m}}}{\lambda_{\mathrm{f}}}\left(1+\phi-\phi^{2 / 3}\right)\right\}\right]$

Effective medium theory

$\phi_{\mathrm{m}} \frac{\lambda_{\mathrm{m}}-\lambda_{\mathrm{eff}}}{\lambda_{\mathrm{m}}+2 \lambda_{\text {eff }}}+\phi_{\mathrm{f}} \frac{\lambda_{\mathrm{f}}-\lambda_{\text {eff }}}{\lambda_{\mathrm{f}}+2 \lambda_{\text {eff }}}=0$

Bruggeman (1935)

$(1-\phi)=\frac{\left(\lambda_{\mathrm{f}}-\lambda_{\text {eff }}\right)\left(\lambda_{\mathrm{m}} / \lambda_{\text {eff }}\right)^{1 / 3}}{\left(\lambda_{\mathrm{f}}-\lambda_{\mathrm{m}}\right)}$

Lewis and Nielsen (1970)

$\lambda_{\mathrm{eff}}=\lambda_{\mathrm{m}} \frac{1+A \cdot B \cdot \phi}{1-B \cdot \phi \cdot \psi}$,

where, $A=\left(K_{\mathrm{E}}-1\right) \quad B=\frac{\lambda_{\mathrm{f}} / \lambda_{\mathrm{m}}-1}{\lambda_{\mathrm{f}} / \lambda_{\mathrm{m}}+A} \quad$ and $\quad \psi=1+\frac{1-\phi_{\mathrm{m}}}{\phi_{\mathrm{m}}^{2}} \phi$

$\frac{1}{\lambda_{\text {eff }}}=\frac{1}{\sqrt{C\left(\lambda_{\mathrm{f}}-\lambda_{\mathrm{m}}\right)\left(B\left(\lambda_{\mathrm{f}}-\lambda_{\mathrm{m}}\right)\right)}} \ln \frac{\sqrt{\lambda_{\mathrm{m}}+B\left(\lambda_{\mathrm{f}}-\lambda_{\mathrm{m}}\right)}+B / 2 \sqrt{C\left(\lambda_{\mathrm{f}}-\lambda_{\mathrm{m}}\right)}}{\sqrt{\lambda_{\mathrm{m}}+B\left(\lambda_{\mathrm{f}}-\lambda_{\mathrm{m}}\right)}-B / 2 \sqrt{C\left(\lambda_{\mathrm{f}}-\lambda_{\mathrm{m}}\right)}}+\frac{1-B}{\lambda_{\mathrm{m}}}$

Cheng and Vachon (1990)

where, $B=\sqrt{3 \phi / 2}$ and $C=-4 \sqrt{2 / 3 \phi}$

$\lambda_{\text {eff }}, \lambda_{\mathrm{m}}$ and $\lambda_{\mathrm{f}}$ are the thermal conductivity of the composites, polymer and filler, respectively. $\phi$ is the concentration of the added filler; $\phi_{\mathrm{m}}$ is the maximum packing fraction of the dispersed particles, $K_{\mathrm{E}}$ is Einstein constant. 
filler loading where continuous filler networks/chains are more likely to be formed.

In view of the above, some researchers (Mamunya et al 1995, 2002; Pabst and Gregorova 2006; Liang et al 2008; Tian and Yang 2008) considered percolation as a more appropriate base for theoretical prediction of the effective thermal conductivity of filled composites with consideration of filler shape and its distribution. However, several investigations on polymer composites with dispersed fillers, also reported absence of percolation behaviour in thermal conductivity with increasing filler content (Sundstrom et al 1972; Kusy and Corneliussen 1975; Progelhof et al 1976; Bigg 1995). It may be noted that in contrast to the electrical conductivity, the concentration dependence of thermal conductivity shows no sudden jump in the percolation threshold region. This is because of the fact that thermal conductivity of the dispersed filler is comparable to that of polymer matrix, while the filler electrical conductivity is $\sim 10^{10}$ times higher than that of polymer matrix. Classical percolation theory (Kutcherov et al 1991) suggests that the effective thermal conductivity of composites relates to the volume fraction of the fillers as:

$$
\lambda_{\text {eff }} \propto \lambda_{\mathrm{f}}\left(\phi-\phi_{\mathrm{c}}\right)^{n},
$$

where $\lambda_{\mathrm{f}}$ is the thermal conductivity of filler, $n$ the percolation exponent, $\phi_{\mathrm{c}}$ and $\phi$ are percolation threshold and volume fraction of fillers, respectively. Mamunya et al (2002) derived effective thermal conductivity of filled polymer composites as:

$$
\log \lambda_{\text {eff }}=\log \lambda_{\mathrm{m}}+\left(\log \lambda_{\mathrm{T}}-\log \lambda_{\mathrm{m}}\right)(\phi / F)^{N},
$$

where $\lambda_{\mathrm{m}}$ is thermal conductivity of matrix, $\lambda_{\mathrm{T}}$ the thermal conductivity of the composite when filler concentration reaches a limited value, $F$ the packing factor and $N$ is an index related to the filler shape, size etc. Considering the percolation similarity between the electrical conductivity and thermal conductivity of particulate filled polymer composites and taking the percolation factor as:

$$
\left[(1-\phi) /\left(1-\phi_{\mathrm{C}}\right)\right]^{n}
$$

Zhang et al (2009) derived the effective thermal conductivity of polymer composites as:

$$
\lambda_{\text {eff }}=\lambda_{\mathrm{f}}\left(\lambda_{\mathrm{c}} / \lambda_{\mathrm{f}}\right)^{\left[\frac{1-\phi}{1-\phi_{\mathrm{C}}}\right]^{\mathrm{n}}},
$$

where $\lambda_{\mathrm{c}}$ is the thermal conductivity of the composite at percolation threshold.

In addition to the above, several researchers used multifractal models to explain percolation conductivity of random composite clusters (Mandelbrot and Given 1984; Blumenfeld et al 1986). Concept developed by Mandelbrot and Given (1984) is used to describe a material microstructure that is not Euclidean, but with a non-integral, so called fractal dimension. Because the fractal dimension could reflect the connectiveness of the fillers, it could be correlated to the probability of the formation of electrically conductive networks in the composite at various fillers fraction. Subse- quently, several fractal based models for both electrical conductivity and effective thermal conductivity of composites were proposed (Putten et al 1992; Zhang et al 1995; Feng et al 2004; Ma et al 2004; Dong et al 2010; Wu et al 2010; Monajjemi et al 2011).

\section{Experimental}

\subsection{Composite preparation}

$\mathrm{La}_{0.7} \mathrm{Ba}_{0.15} \mathrm{Sr}_{0.15} \mathrm{MnO}_{3}$ (LBSMO) was prepared by a soft chemical process, known as 'pyrophoric technique'. The pyrophoric synthesis route was basically a chemical method, which produced fine grained (nano-sized powder) by embedding the metal-ion complex in the mass of organic matrix (Das and Dey 2006). The effectiveness of this method lies in the homogeneity and sinterability of the powder at relative temperature to get the desired phase as well as energy saving with respect to the conventional solid state route. The starting materials for the synthesis of the present sample were: $\mathrm{La}_{2} \mathrm{O}_{3}, \mathrm{Ba}\left(\mathrm{NO}_{3}\right)_{2}, \mathrm{Sr}\left(\mathrm{NO}_{3}\right)_{2}$ and $\left(\mathrm{CH}_{3} \cdot \mathrm{COO}\right)_{2} \mathrm{Mn} \cdot 4 \mathrm{H}_{2} \mathrm{O}$ of purity $99.9 \%$. Initially, the measured amount of $\mathrm{La}_{2} \mathrm{O}_{3}, \mathrm{Ba}\left(\mathrm{NO}_{3}\right)_{2}$ and $\mathrm{Sr}\left(\mathrm{NO}_{3}\right)_{2}$ were separately dissolved in minimum amounts of dilute nitric acid and de-ionized water, respectively to prepare metal nitrate stock solutions $(0.01 \mathrm{M})$. Separately aqueous solutions of $\left(\mathrm{CH}_{3} \cdot \mathrm{COO}\right)_{2} \mathrm{Mn} \cdot 4 \mathrm{H}_{2} \mathrm{O}(0 \cdot 01 \mathrm{M})$ was prepared by dissolving stoichiometric amounts of the salt in de-ionized water. Appropriate amounts of the respective metal nitrate solutions were mixed with triethanolamine (TEA). The amount of TEA was maintained at 2 moles per unit mole of the metal ions. Stiochiometric amount of the aqueous solution of $\left(\mathrm{CH}_{3} \cdot \mathrm{COO}\right)_{2} \mathrm{Mn} \cdot 4 \mathrm{H}_{2} \mathrm{O}$ was then added under hot condition $\left(80^{\circ} \mathrm{C}\right)$ with continuous stirring. The $\mathrm{pH}$ of the entire solution was maintained between 5 and 6 by adding TEA and the final volume of the solution was made $100 \mathrm{cc}$ by adding de-ionized water. An aqueous solution of sucrose (4 moles per unit mole of the metal ion) and PVA (10 mole \% with respect to the total moles of the cations) were added to the above clear solution of metal ion complexes under hot condition to obtain the homogeneous polymeric-based precursor solutions. The solution was then slowly heated to $\sim 200^{\circ} \mathrm{C}$ with continuous stirring and eventually evaporated to dryness. At this stage, vigorous pyrophoric reaction took place and finally resulted into a fluffy charred organic mass. This fluffy organic mass was allowed to burn for $\sim 2 \mathrm{~h}$ till it became completely dry. The fully charred organic mass contained the desired metal ions embedded in its matrix. Subsequently, the carbon rich mass was crushed and was calcined at $800^{\circ} \mathrm{C}(20 \mathrm{~h})$ to get carbon-free, nano-sized powders of the desired composition. The powder thus obtained was sintered at $1100^{\circ} \mathrm{C}$ in air for $24 \mathrm{~h}$ and then furnace cooled to room temperature. The sintered pellets were again crushed into powder, which was used for making the composites samples required for the present investigation. XRD of $\mathrm{La}_{0.7} \mathrm{Ba}_{0.15} \mathrm{Sr}_{0.15} \mathrm{MnO}_{3}$ powder shown in figure 1 , confirmed the single phase nature and 
rhombohedral lattice structure with space group, $R \overline{3} C$, in which $\mathrm{La} / \mathrm{Ba} / \mathrm{Sr}$ atoms were at $6 \mathrm{a}(0,0,1 / 4)$ positions, $\mathrm{Mn}$ at $6 \mathrm{~b}(0,0,1 / 2)$ and $\mathrm{O}$ at $18 \mathrm{e}(x, 0,1 / 4)$ position. The structural parameters $(a=b=5.4977 \AA, c=13.5253 \AA$ ) were obtained by refining the experimental data using Rietveld FULLPROF program. Crystallites size obtained from the

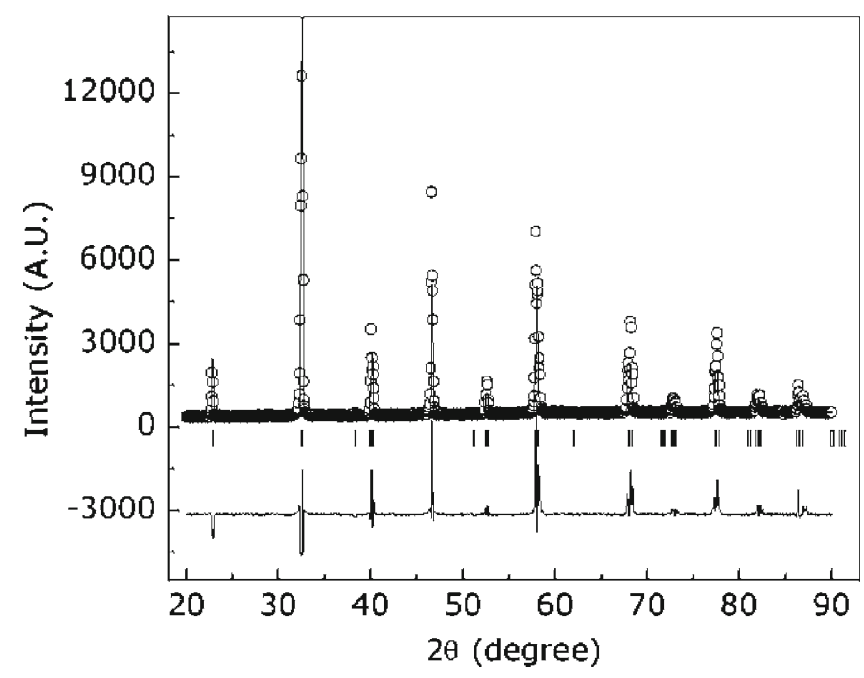

Figure 1. X-ray diffraction pattern of prepared $\mathrm{La}_{0.7} \mathrm{Ba}_{0 \cdot 15} \mathrm{Sr}_{0.15}$ $\mathrm{MnO}_{3}$ powder.
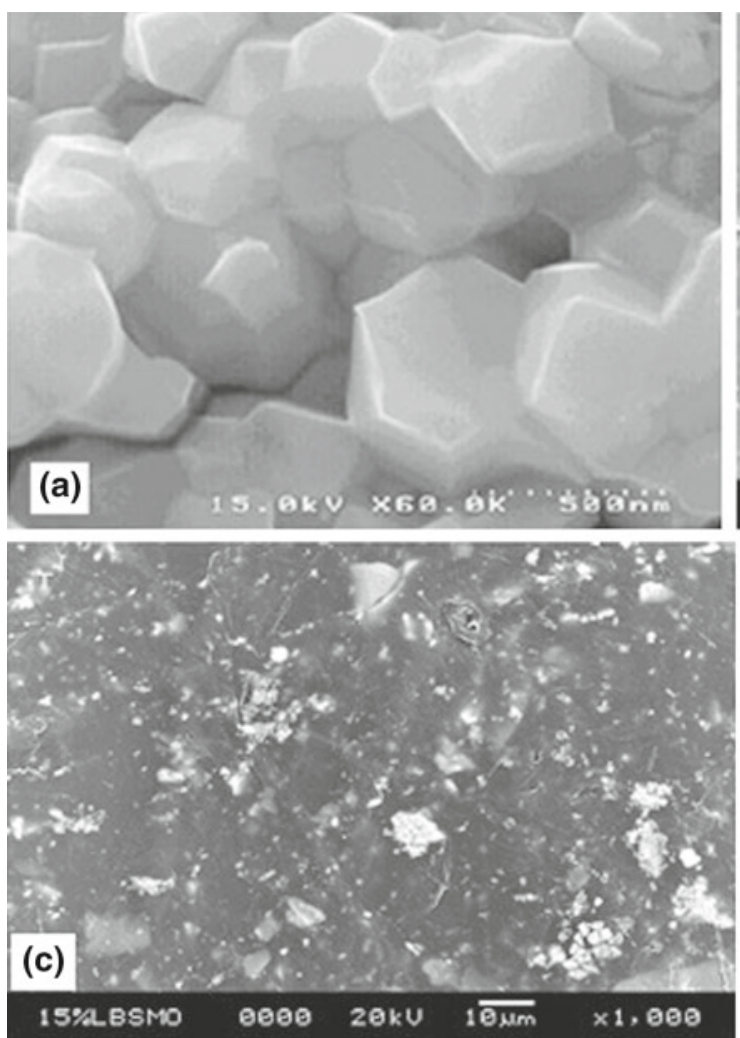

XRD data was $\sim 24.3 \mathrm{~nm}$. The Curie temperature $\left(T_{\mathrm{C}}\right)$ of $\mathrm{La}_{0.7} \mathrm{Ba}_{0.15} \mathrm{Sr}_{0.15} \mathrm{MnO}_{3}$ obtained from a.c. susceptibility measurement was $74.8^{\circ} \mathrm{C}$. SEM photograph of LBSMO powder (figure 2a) shows the particles to be primarily of irregular shapes.

For the preparation of composites, various volume fractions $(0-0 \cdot 30)$ of LBSMO powder was mixed thoroughly with HDPE powder (total mass, $\sim 20 \mathrm{~g}$ ). $50 \mathrm{ml}$ of xylene was added to the mixture and slowly heated to $\sim 80^{\circ} \mathrm{C}$ with vigorous stirring till xylene completely evaporated. The homogeneous mixture of HDPE/LBSMO was then transferred in a stainless steel die and molded in pellet form in a hot press at $120^{\circ} \mathrm{C}$ under a pressure of $3 \mathrm{~T} / \mathrm{cm}^{2}$ and was then slowly cooled to room temperature. SEM pictures of the composites revealed non-spherical and irregular shaped LBSMO particles which were distributed fairly uniformly in HDPE matrix (figures $2 \mathrm{~b}-\mathrm{d}$ ). Presence of clusters and percolation paths was also clearly seen, particularly in composites prepared with higher volume fraction of $\mathrm{La}_{0.7} \mathrm{Ba}_{0.15} \mathrm{Sr}_{0.15} \mathrm{MnO}_{3}$ filler.

\subsection{Thermal conductivity}

Most of the techniques used for determination of thermal conductivity suffered from a few limitations, viz. timeconsuming, expensive to set up, low accuracy, or limited measurement range of thermal conductivity. Transient plane

Figure 2. (a) Scanning electron micrograph of $\mathrm{La}_{0.7} \mathrm{Ba}_{0.15} \mathrm{Sr}_{0 \cdot 15} \mathrm{MnO}_{3}$ (LBSMO) powder and (b)-(d) typical scanning electron micrographs of prepared HDPE/LBSMO composites containing 0.03, 0.15 and 0.3 volume fraction of LBSMO filler. 
source (TPS) method (Gustavsson et al 1994) was found superior to the common techniques for determining thermal conductivity and this method does not suffer from the limitations mentioned above. The present investigation was, therefore, carried out using TPS technique employing a hot disc sensor (M/s Disk AB, Sweden). A hot disc sensor was sandwiched between two identical samples and a constant current was passed through the hot disc (figure 3a). The average temperature increase of the sensor was

$$
\overline{\Delta T(\tau)}=\frac{P_{0}}{\pi^{3 / 2} a \lambda} D_{\mathrm{S}}(\tau),
$$

where $P_{0}$ is the output power to the sensor, $a$ the radius of the largest ring in the hot disk, $\lambda$ the thermal conductivity of the sample, and $\tau=\sqrt{\mu t} / a$ the characteristic time ratio, $\mu$ the thermal diffusivity of the sample, and $t$ the time.

The function, $D_{\mathrm{s}}(\tau)$, was

$$
\begin{aligned}
& D_{\mathrm{S}}(\tau) \\
& =[m(m+1)]^{-2} \\
& \times \int_{0}^{\tau} \frac{\mathrm{d} \sigma}{\sigma^{2}}\left[\sum _ { l = 1 } ^ { m } l \left\{\sum_{p=1}^{m} p \cdot \exp \left(\frac{\left(-l^{2}+p^{2}\right)}{2 \sigma^{2} m^{2}}\right)\right.\right. \\
& \left.\left.\times I_{0}\left(\frac{l p}{2 \sigma^{2} m^{2}}\right)\right\}\right]
\end{aligned}
$$

$I_{0}$ is the modified Bessel function. Knowing the relation between $t$ and $\tau$, a linear plot between $\Delta T$ and $D_{\mathrm{S}}(\tau)$ is obtained and thermal conductivity $(\lambda)$ is calculated from the slope equation (9). Bohac et al (2000) investigated the basic criterion for selecting experimental times in thermal conductivity measurements using the hot disk technique. The optimal 'time window' for a single transient recording was estimated from:

$$
t_{\min } \geq\left(\delta_{\text {ins }}\right)^{2} / \mu_{\text {ins }}
$$

and

$$
0 \cdot 3 \leq\left(t_{\max } / \theta\right) \geq 1 \cdot 1
$$

with

$$
\theta=\frac{a^{2}}{\mu} .
$$

$\delta_{\text {ins }}$ and $\mu_{\text {ins }}$ are the insulation thickness and thermal diffusivity of insulation material, respectively. The probing depth calculated for the sample dimension and the hot disc sensor combination was $\sim 4 \mathrm{~mm}$ and was well within the dimensions of the samples used $(\phi=25 \mathrm{~mm}$, thickness $=10 \mathrm{~mm})$. Therefore, the influence of sample boundaries on the measured data was negligible. Experiments were performed
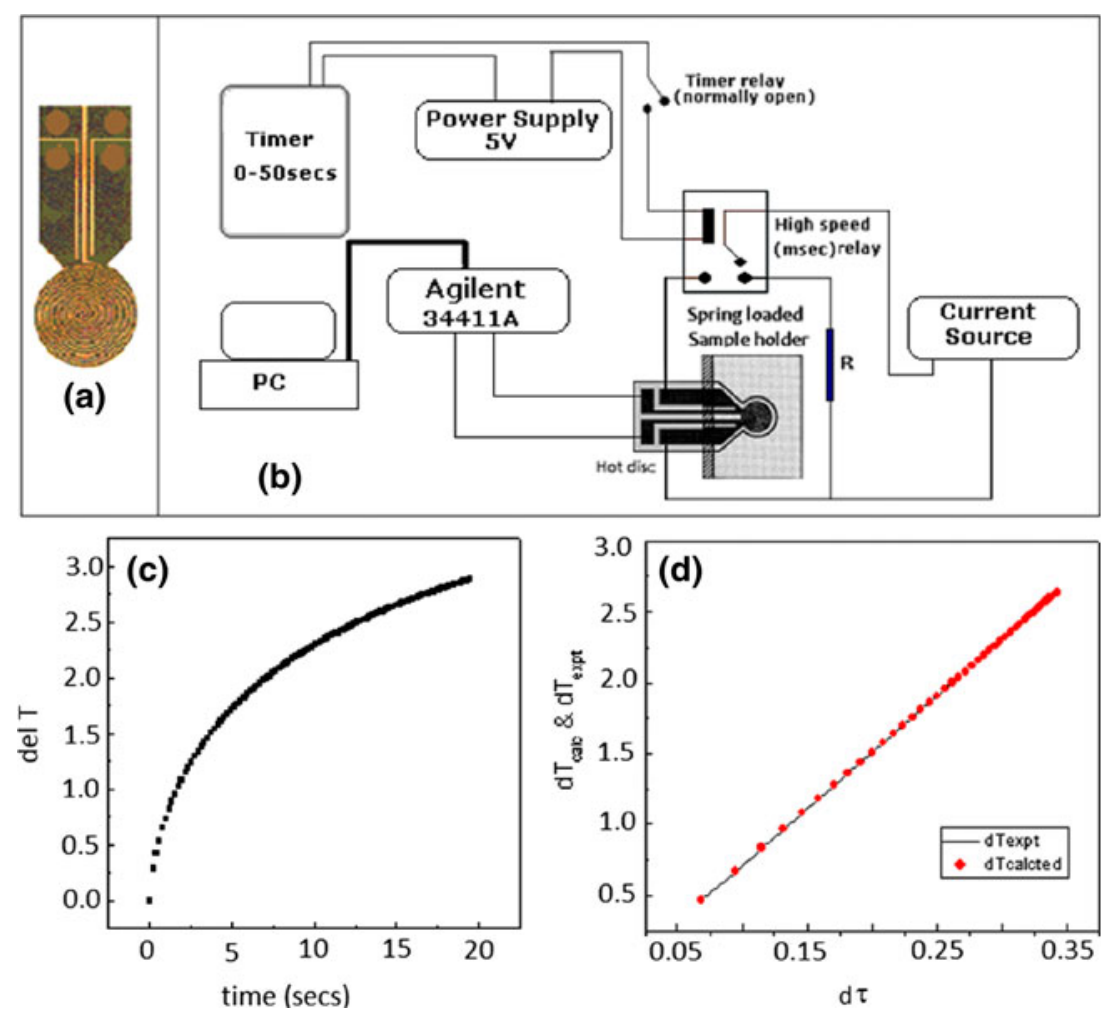

Figure 3. (a) Typical hot disc sensor used in present experiments for thermal conductivity measurement, (b) schematic of the electrical circuit for measuring thermal conductivity of composites, (c) typical response of change of temperature vs time and (d) points are experimentally determined $(\Delta T)$ as a function of $D(\tau)$ for a typical composite sample based on typical results of $\Delta T$ vs time. The straight line is linear fit of data. 
using two identical and polished samples of the same composition. The hot disc sensor $(\phi=3.322 \mathrm{~mm})$ was sandwiched firmly in between the two samples under spring load arrangement, such that very good thermal contact between the samples and the sensor was maintained. The applied power to the hot disk sensor used was between 0.05 and $0.15 \mathrm{~W}$. The change of sensor voltage was recorded by a PC controlled high speed DMM (Agilent 34411A). Schematic of the experimental arrangement is shown in figure $3 \mathrm{~b}$. Figure $3 \mathrm{c}$ shows a typical plot of the temperature rise of the sensor as a function of time during thermal conductivity measurement for the present composite, and figure $3 \mathrm{~d}$ shows measured $\Delta T$ as a function of $D(\tau)$. It may be noted that while computing the $\Delta T$ vs $D(\tau)$ curve, the first 8-10 points are excluded from the raw data, in order to nullify influence of the insulating layer of the sensor. Thermal conductivity was finally obtained from the slope of $\Delta T$ vs $D(\tau)$ plot. It is to be noted that at each temperature, thermal conductivity of the composites were measured five times and their average value taken
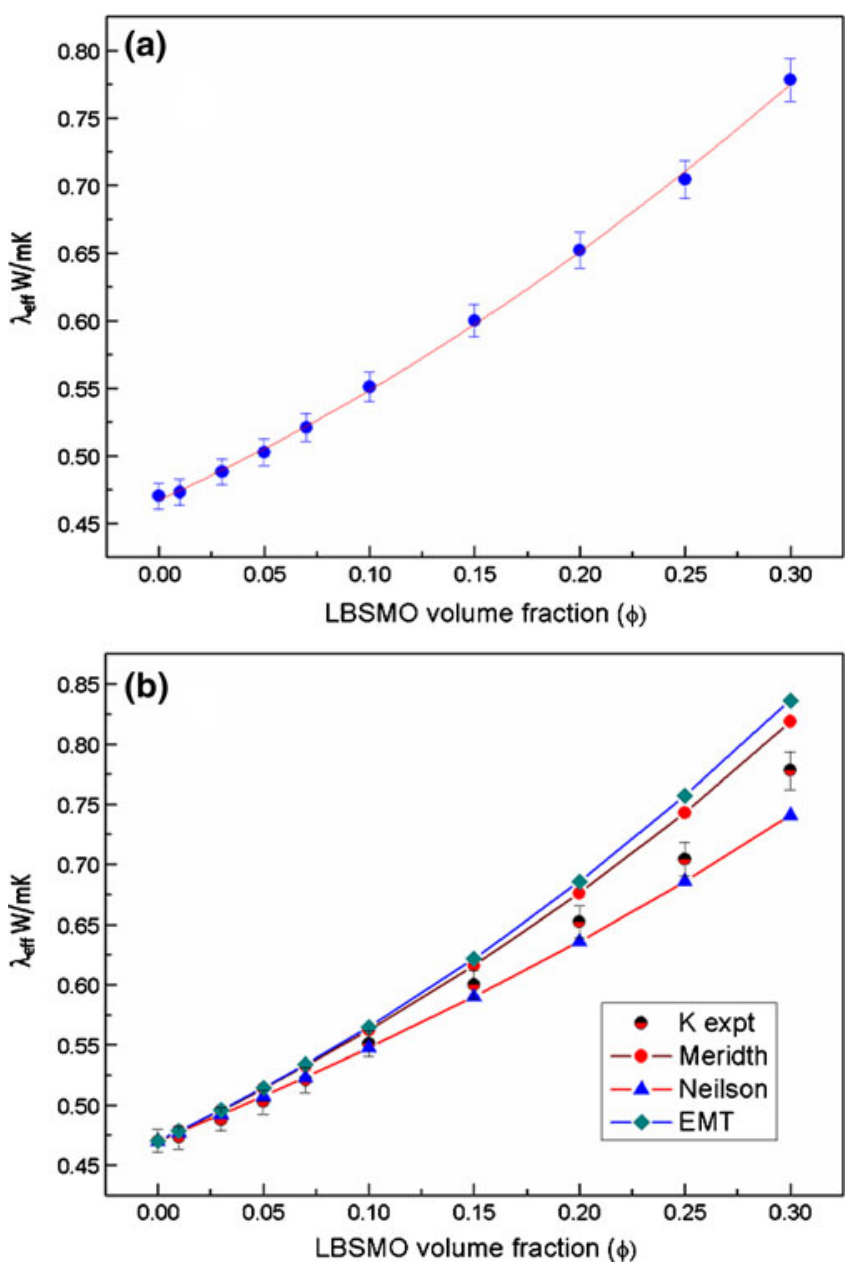

Figure 4. (a) Effective thermal conductivity of HDPE/LBSMO composites at room temperature as a function of LBSMO volume fraction and (b) comparison of measured effective thermal conductivity of HDPE/LBSMO composites to those estimated by various models. as $\lambda_{\text {eff. }}$. The overall maximum uncertainty in our thermal conductivity measurement was estimated to be $\sim 3 \%$.

\section{Results and discussion}

Figure $4 \mathrm{a}$ shows measured room temperature effective thermal conductivity of HDPE/LBSMO composites as a function of LBSMO filler concentration. The thermal conductivity of the composites enhances by $\sim 65 \%$ to that for pure HDPE for 0.30 volume fraction of LBSMO. The enhancement in the thermal conductivity of HDPE/LBSMO composites is expected, as the thermal conductivity of the filler (LBSMO) is nearly five times higher than that for HDPE. It may be noted that with increase in volume fraction
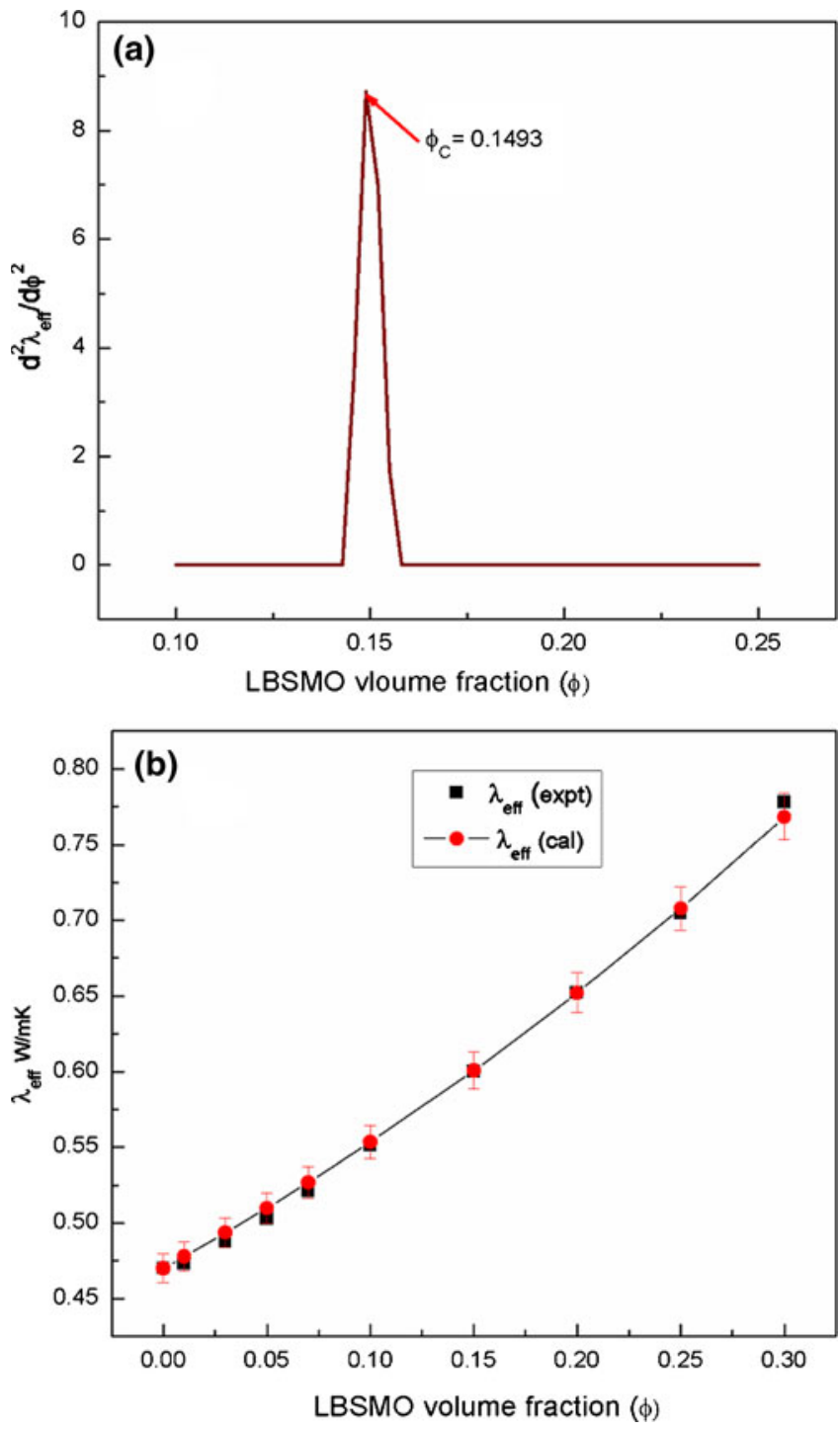

Figure 5. (a) Plot of double derivative of measured effective thermal conductivity, $\lambda_{\text {eff }}$ vs volume fraction $(\phi)$ for determination of percolation threshold $(\phi)$ and (b) comparison of measured effective thermal conductivity of HDPE/LBSMO composites ( $\left.\lambda_{\text {eff }}\right)$ to those predicted by percolation models (8). 
of LBSMO in HDPE, interaction between LBSMO particles increases and they come in contact with each other (as is evident from SEM). This results in the ease in transfer of heat and consequent enhancement of the effective thermal conductivity of the composites.

The measured effective thermal conductivity $\left(\lambda_{\text {eff }}\right)$ of the HDPE/LBSMO composites has been examined vis-àvis the predictions based on models referred above (figure $4 \mathrm{~b}$ ). In figure $4 \mathrm{~b}$, the calculated thermal conductivity of HDPE/LBSMO composites is shown only for those models, that deviates from the measured values by $<15 \%$. It may be mentioned that $\lambda_{\text {eff }}$ expressions derived by Bruggeman (1935), Russell (1935) and Cheng and Vachon (1990) fail to predict the effective thermal conductivity of the present composites, viz. Russell's expression (2) largely overestimates, while that derived by Bruggeman (1935) severely underestimates the measured thermal conductivity of HDPE/LBSMO composites. Failure of Cheng and Vachon (1990) model is probably due to the fact that this model is derived for composites with $\frac{\lambda_{\mathrm{f}}}{\lambda_{\mathrm{m}}}>100$, which in the present case is not satisfied. Figure $4 \mathrm{~b}$ shows that Meredith and Tobias (1962) model predicts fairly well the effectiveness ( $\left.\lambda_{\text {eff }}\right)$ of the composites, only for low LBSMO volume fraction $(<0 \cdot 08)$. Meredith's expression (1) though was derived for the thermal conductivity of composites containing high thermal conductivity fillers and for higher filler content, the predicted values in the present case, is $\sim 5 \%$ higher than the measured data for composites with LBSMO loading of $>0 \cdot 1$. The effective thermal conductivity of HDPE/LBSMO composites calculated for randomly packed irregularly shaped particles $(A=3$ and $\left.\varphi_{\mathrm{m}}=0.74\right)$ using equation of Lewis and Nielsen (1970), fairly agrees with the measured data only for low filler content. He et al (2007) also reported that for epoxy $/ \mathrm{Si}_{3} \mathrm{~N}_{4}$ composites, Lewis model works only for low filler concentration and with increasing $\mathrm{Si}_{3} \mathrm{~N}_{4}$ volume fraction, the calculated values starts to deviate appreciably from the experimental data. Further, the constants $A$ and $B$ in (5) being empirical, getting their exact values are difficult (Progelhof et al 1976; Hui et al 1999). Similarly, the EMT model also fails to give an acceptable prediction of the thermal conductivity at high volume fraction of the filler (figure $4 b$ ). Thus, it is seen that all the above models lack in the general validity to predict thermal conductivity of HDPE/LBSMO composites over the studied volume fraction range of the filler.

It may be noted that the percolation based model proposed by Zhang et al (2009) predicts that the thermal conductivity percolation threshold appears only if the ratio of the electrical conductivity of the filler to the polymer electrical conductivity is larger than $\sim 10^{5}$, which in the present case is satisfied. In addition, as noted before, SEM photographs of HDPE/LBSMO composites also show the formation of overlapping clusters/percolation paths, particularly at high filler loading. In view of the above, it is felt reasonable to evaluate $\left(\lambda_{\text {eff }}\right)$ in terms of the model proposed by Zhang et al (2009) and confirm the role of percolation in the enhanced thermal conductivity observed for our composites containing LBSMO filler particles.

Generally, it is observed that the measured thermal conductivity of the composites increases fairly rapidly for filler volume fraction between $0 \cdot 15$ and $0 \cdot 20$. The filler volume fraction at which the effective thermal conductivity of the composites starts to increase rapidly is usually taken as the percolation threshold $\left(\phi_{\mathrm{C}}\right)$. The percolation threshold $\left(\phi_{\mathrm{C}}\right)$ in our case, is determined by plotting the double-derivative of the measured effective thermal conductivity for filler volume fraction between $0 \cdot 10$ and $0 \cdot 20$. The filler volume fraction corresponding to which $\left(\frac{d^{2} \lambda_{\text {eff }}}{d \phi^{2}}\right)$ shows maximum is taken as the percolation threshold $\left(\phi_{\mathrm{C}}\right)$. Plot of $\left(\frac{d^{2} \lambda_{\text {eff }}}{d \phi^{2}}\right)$ vs $\phi$ for HDPE/LBSMO composites is shown in figure 5a and the percolation threshold volume fraction $\left(\phi_{\mathrm{C}}\right)$ is determined to be $0 \cdot 1493$. The observed percolation threshold of 0.1493 in the present case is comparable to the recently reported study on manganite nanoparticles in PVDF matrix (Chandrasekhar et al 2009). Percolation in general depends on the particle size (Deepa et al 2007) and the method of preparation that modifies the matrix-filler interface. The deviation of percolation threshold from 0.3 as expected from the mean field approximation is due to the nanometric size of the manganite particles, where a small amount of nanoparticles is sufficient to trigger the conducting path (Wang and

Table 2. Comparison of measured effective thermal conductivity of HDPE-LBSMO composites at $30^{\circ} \mathrm{C}$ with those estimated by various models.

\begin{tabular}{llllll}
\hline $\begin{array}{l}\text { LBSMO volume } \\
\text { fraction }\end{array}$ & $\begin{array}{c}\lambda_{\text {eff }}(\text { expt.) } \\
(\mathrm{W} / \mathrm{mK})\end{array}$ & $\begin{array}{c}\text { Percolation } \\
(\mathrm{W} / \mathrm{mK})\end{array}$ & $\begin{array}{c}\text { Meredith } \\
(\mathrm{W} / \mathrm{mK})\end{array}$ & $\begin{array}{c}\text { Lewis } \\
(\mathrm{W} / \mathrm{mK})\end{array}$ & $\begin{array}{c}\text { EMT } \\
(\mathrm{W} / \mathrm{mK})\end{array}$ \\
\hline 0 & 0.47 & 0.46999 & 0.47 & 0.47 & 0.47 \\
0.01 & 0.473 & 0.47773 & 0.4784 & 0.47736 & 0.47843 \\
$0 \cdot 03$ & 0.4881 & 0.49359 & 0.49572 & 0.4923 & 0.49595 \\
$0 \cdot 05$ & 0.5027 & 0.50999 & 0.51375 & 0.50758 & 0.51439 \\
$0 \cdot 07$ & 0.521 & 0.52694 & 0.53253 & 0.52321 & 0.53378 \\
$0 \cdot 1$ & 0.551 & 0.55345 & 0.56219 & 0.5474 & 0.56476 \\
0.15 & 0.600 & 0.60067 & 0.61599 & 0.58998 & 0.62172 \\
$0 \cdot 2$ & 0.6522 & 0.652 & 0.67598 & 0.63589 & 0.68574 \\
0.25 & 0.7044 & 0.7078 & 0.74311 & 0.68584 & 0.75717 \\
0.3 & 0.778 & 0.76847 & 0.81859 & 0.74066 & 0.83614 \\
\hline
\end{tabular}


Dang 2005; Li et al 2006; Dang et al 2007; Yao et al 2007; He et al 2009).

The composites thermal conductivity $\left(\lambda_{\mathrm{C}}\right)$ at percolation threshold $\left(\phi_{\mathrm{C}}\right)$ is $0.60 \mathrm{~W} / \mathrm{mK}$. As can be seen from (8), the effective thermal conductivity ( $\lambda_{\text {eff }}$ ) reduces to that for the matrix (HDPE) for filler volume fraction $\phi=0$ and using the values of $\phi_{\mathrm{c}}, \lambda_{\mathrm{c}}, \lambda_{\mathrm{f}}$ and $\lambda_{\mathrm{m}}$, the percolation exponent $(n)$ is calculated to be 0.9768 . Finally, incorporating the values of $\phi_{\mathrm{c}}, \lambda_{c}$ and $n$ in (8), ( $\left.\lambda_{\text {eff }}\right)$ for HDPE/LBSMO composites is calculated (table 2) and plotted in figure 5b. It is evident from figure $5 \mathrm{~b}$, that compared to all other models, the percolation based model proposed by Zhang et al (2009) predicts extremely well $(\sim 1 \%)$ the effective thermal conductivity $\left(\lambda_{\text {eff }}\right)$ of HDPE/LBSMO composites over the entire measured range of filler volume fraction. The validity of the percolation based thermal conductivity model (Zhang et al 2009) indicates that the observed rapid enhancement in the effective thermal conductivity $\left(\lambda_{\text {eff }}\right)$ of $\mathrm{HDPE} / \mathrm{La}_{0.7} \mathrm{Ba}_{0.15} \mathrm{Sr}_{0.15} \mathrm{MnO}_{3}$ composites at high volume fraction of the filler corresponds to the formation of percolation network between the fillers in the polymer matrix. It is also important to note that, unlike all other models, (8) derived by Zhang et al (2009), does not involve any empirical/adjustable parameters and all the quantities are extracted from the experimental data.

\section{Conclusions}

Effective thermal conductivity of HDPE/LBSMO composites as a function of filler (LBSMO) volume fraction between 0 and 0.3 is measured. Effective thermal conductivity of the composites increases with increasing LBSMO content and for 0.3 volume fraction of LBSMO, it enhances by $\sim 65 \%$ compared to that for pure HDPE. The results have been discussed employing various thermal conductivity models, including effective medium theory, Meredith model and Lewis model. While all these models can predict fairly well the measured data for low filler loading $(<0.08)$, large deviations from the measured values are observed for filler volume fraction $>0 \cdot 1$. This is because all these models are based on the assumption that the fillers are homogeneously dispersed in the matrix and do not consider formation of percolative paths or, channels of conducting LBSMO fillers even for higher filler volume fractions. We confirm that Zhang's model based on percolation theory gives a remarkable prediction of the measured effective thermal conductivity of HDPE/LBSMO composites. At high volume fraction of the filler and depending on their actual distribution in polymer, the conducting fillers may form percolation networks in the polymer matrix. In such a situation, the rapid increase in effective thermal conductivity of polymer composites can be accounted well by the model based on percolation theory. However, when the characteristic size of the particles in the nanocomposites is comparable to or smaller than the phonon mean free path, the phonon scattering at interfaces between two materials can introduce significant thermal resistance in the highly conductive phonon pathway. Such interfacial thermal resistance can reduce the thermal conductivity of the nanoparticle composites. The thermal conductivity of the random nanoparticle composites may, therefore deviate significantly from the predictions of the percolation theory. Further studies along this direction are needed.

\section{Acknowledgement}

One of the authors (TKD) acknowledges CSIR, New Delhi, for financial support in the form of a research project.

\section{References}

Agari Y and Uno T 1986 J. Appl. Polym. Sci. 325705

Battabyal M and Dey T K 2006 Physica B373 46

Bigg D M 1995 Adv. Polym. Sci. 1191

Blumenfeld R, Meir Y, Harris A B and Aharony A 1986 J. Phys. A19 L791

Bohac V, Gustavsson M K, Kubikar L and Gustafsson S E 2000 Rev. Sci. Instrum. 712452

Bruggeman D A G 1935 Am. Phys. 24636

Carson J K, Lovatt S L, Tanner D J and Cleland A C 2005 Int. J. Heat and Mass Transf. $\mathbf{4 8} 2150$

Chandrasekhar K D, Venimadhav A and Das A K 2009 Appl. Phys. Letts 95062904

Cheng S C and Vachon R L 1990 Int. J. Heat Mass Trans. 11537

Cohn J L 2000 J. Supercond.: Incorp. Novel Magn. 13291

Dang Z M, Wang L, Yin Y, Zhang Q and Lei Q Q 2007 Adv. Mater. (Germany: Weinheim) 19852

Das S and Dey T K 2006 J. Phys. Condens. Matter 187629

Deepa K S, Sebastian M T and James J 2007 Appl. Phys. Letts 91 202904

Dong X J, Hu Y F, Wu Y Y, Zhao J and Wan Z Z 2010 Chin. Phys. Lett. 27044401

Feng Y, Yu B, Zou M and Zhang D 2004 J. Phys. D: Appl. Phys. 37 3030

Fujishiro H and Ikebe M 1999 Physica B263-264 691

Goyal R K, Tiwari A N, Mulik U P and Negi Y S 2007 Compos. Sci. Technol. 671802

Gustavsson M, Karawacki E and Gustafsson S E 1994 Rev. Sci. Instrum. $\mathbf{6 5} 3856$

Hakansson B and Ross R G 1990 J. Appl. Phys. 683285

Hamilton R L and Crosser O K 1962 Ind. Eng. Chem. Fund. 1187

He H, Renli F, Han Y, Shen Y and Song X 2007 J. Mater. Sci. 42 6749

He F, Lau S, Chan H L and Fan J 2009 Adv. Mater. (Germany: Weinheim) 21710

Hejtmanek J, Jirak Z and Marysko M 1999 Phys. Rev. B60 14057

Hui P M, Zhang X, Markworth A and Stroud D 1999 J. Mater. Sci. 345497

Keblinski P, Phillpot S R, Choi S U S and Eastman J A 2002 Int. J. Heat Mass Trans. $\mathbf{4 5} 855$

Kumlutas D, Tavman I H and Coban M T 2003 Compos. Sci. Technol. 63113

Kusy R P and Corneliussen R D 1975 Polym. Eng. Sci. 15107

Kutcherov V, Hakansson B, Ross G and Backstrom G 1991 J. Appl. Phys. 711732

Lewis T and Nielsen L 1970 J. Appl. Polym. Sci. 141449 
Liang L H, Wei Y G and Li B 2008 J. Phys.: Condens. Matter 20 365201

Li Y, Xu M, Feng J Q and Dang Z M 2006 Appl. Phys. Letts 89 072902

Li Y, Zhou J, Tung S, Schneider E and Xi S 2009 Powder Technol. 19689

Ma Y, Yu B, Zhang D and Zou M 2004 J. Appl. Phys. 95 6426

Mamunya Y P, Davydenko V V and Lebedev E V 1995 Polym. Compos. 16319

Mamunya Y P, Davydenko V V, Pissis P and Lebedev E V 2002 Euro. Polym. J. 381887

Mandelbrot B B and Given J A 1984 Phys. Rev. Lett. 521853

Maxwell J C 1954 A treatise on elect. and magn. (NY: Dover Pub, Inc.) 3rd ed.

Meredith R E and Tobias C W 1962 Adv. in Electrochem and Electrochemical Engg. (New York: Wiley Interscience) 215

Monajjemi M, Baheri H and Mollaamin F 2011 J. Struct. Chem. 52 54

Murshed S M S, Leong K C and Yang C 2008 Appl. Therm. Engg. 282109

Ott H J 1981 Plastic and Rubber Processing and Application 19
Pabst W and Gregorova E 2006 Ceram. Int. 3289

Progelhof R C, Throne J H and Ruetsch R R 1976 Polym. Eng. Sci. 16615

Putten D, Moonen J T, Brom H B, Brokken-Zijp J C M and Michels M A J 1992 Phys. Rev. Letts 69494

Russell H W 1935 J. Am. Ceram. Soc. 181

Sass J H, Lachenbroch H H and Munroe R 1971 Z. Geophys. Res. 763391

Sundstrom D W and Lee Y-D 1972 J. Appl. Polym. Sci. 163159

Tian W and Yang R 2008 CMES 24123

Wang L and Dang Z M 2005 Appl. Phys. Letts 87042903

Wang X Q and Mujumdar A S 2007 Int. J. Therm. Sci. 461

Wong P and Bollampally R S 1999 J. Appl. Polym. Sci. 743396

Wu C, Cho T J, Xu J, Lee D, Yang B and Zachariah M R 2010 Phys. Rev. E81 011406

Xu Y, Chung D D I and Mroz C 2001 Composites Part A 321749

Yao S H, Dang Z M, Jiang M J, Xu H P and Bai J 2007 Appl. Phys. Letts 91212901

Zhang G, Xia Y, Wang H, Tao Y, Tao G, Tu S and Wu H 2009 J. Compos. Mater. (DOI: 10.1177/0021998309349690)

Zhang M Q, Xu J R, Zeng H M, Huo Q, Zhang Z Y and Yun F C 1995 J. Mater. Sci. 304226 